\title{
"HUMANITY" ASPECT OF EVENTUAL USE OF THE NEUTRON WEAPONS
}

\begin{abstract}
The non-proliferation of nuclear weapons is a matter of policy and social responsibility for world peace, while the peaceful development of nuclear technology is a matter of freedom, sovereignty and equality of nations. It is known that the improvement and development of nuclear weapons in the Cold War era was a constant process. In this paper, the author addresses the reasons behind the development and introduction of certain types of nuclear weapons in the armament, which according to the development programs at that time it is classified as weapons with intensive initial nuclear radiation. The neutron weapons emerge in part as a consequence of the doctrines of nuclear countries in accordance with the classification of possible wars that would have taken place in that era. The logical consequence of such a situation was the limited nuclear war, in which the nuclear forces became not only the owners of nuclear weapons, but also its hostages. The vision was that in a match with limited nuclear strikes, the winner might be the side that has more sophisticated weapons at the moment. The neutron bomb, officially defined as a high-radiation weapon, is a low-performance thermonuclear weapon designed to maximize deadly neutron radiation in the immediate vicinity of an explosion while minimizing the physical power of the explosion itself. The question remains whether the spread of various and even partially inaccurate information that protection is very difficult, almost impossible, may have one additional motive for the production and spread of this weapon - to influence morality, the political situation and to exert psychological pressure, not only to armed forces but also the population in general ?!
\end{abstract}

Keywords: NEUTRON WEAPONS, HUMANITY, LIMITED WAR, NUCLEAR ENERGY, RADIATION

\section{Introduction}

Since 1974, the time when neutron weapons were first put on active duty, it has given the basic features on the concept of war and its preparations, primarily on a strategic and, consequently, operational-tactical scale. At the time, the balance of power in nuclear weapons was one of a number of deterrents to World War, increasing the likelihood that strategic weapons would be used only as a last resort, with further deterrence.The post-World War II era is characterized by enormous scientific advances in research, development, and the improvement of nuclear weapons. During that time, the 
world's nuclear potential - with the introduction of new types of nuclear weapons that differ in construction, mode of operation, power and mode of launch - has become more complex and diverse. Until the end of the Cold War, two directions could be distinguished in the development of nuclear weapons: 1) an attempt to produce missiles with operational-strategic purpose and to develop and improve the means for their transmission and launch. The result was that strategic nuclear weapons exceeded the natural limit of weapons in general, that is, it achieved the capacity to destroy humanity, reaching the limit of the absurd - humanity could not use it without causing its own destruction; 2) research, development and refinement of tactical nuclear weapons with small (10 KT) and very small force (1 KT) of fission principles of energy release.

In the development of small and very small power tactical nuclear weapons, three generations can be distinguished. The first generation is the advanced missiles that have already been used. The second generation consists of fission missiles with significantly less force whichstarted to replace some of the first-generation warheads in the early 1980s. The reduction in the strength of the missile has caused significant changes in the effects, so that the effect of the initial radioactive action is enhanced in the fission missiles with a small and especially very small yield. Basically this is a physical process, according to which with increasing force, the mechanical-thermal effect grows faster than the radiation effect, and in a situation of force reduction, the radiation decreasesmore slowly. Additional radioactivity in such missiles is less dangerous, mainly for two reasons: 1) air nuclear blast is the most efficient way of application, because additional contamination from a standstill of direct combat action is practically negligible; 2) even if surface explosions are applied to it, the radioactive cloud is smaller, and thus the volume of contamination is relatively small. In that period (early 80's) the development and further improvement of second-generation missiles were basically aimed at developing missiles with less explosive charge, new construction solutions and materials, which would improve some of the technical-technological features. The third generation in the development of tactical nuclear weapons is small and very small force missiles on the fusion principle of energy release, i.e. nuclear weapons with increased radiation effect, the so-called neutron weapons.

\section{History and controversy of neutron weapons}

The idea of making a neutron weapon is as old as the idea of making a thermonuclear weapon. Even then, it has been observed that by fusing light nuclei, a missile with a thousand times reduced force can be obtained, with a different distribution of the released energy and different pronounced effects of action, which would enable combat application in tactical terms. The materialization of this idea has been going on for quite a long time with variable intensity. The credit for the concept of the neutron bomb goes to Samuel T. Cohen of the Lawrence Livermore National Laboratory, who developed the concept in 
1958 (McFadden, 2010). The initial development was part of the „Dove and Starling" project and was initially tested underground in early 1962. The design of the weapon version was made in 1963 (Cochran et al., 1987, 23). The development of two short-range missile design products for the MGM-52 Lance mobile ground artillery field missile system for the US Army began in July 1964 with the W63 warhead in Liverpool and the W64 warhead in Los Alamos. Once both enter the testing phase in 1964, the W64 will be discontinued in favor of the W63, and later in November 1965 the W63 will be discontinued in favor of the new W70 Mod 0 design.

Until then, the same concept had been used to develop warheads for the Sprint anti-ballistic missile (ABM), with the design of the Livermore W65 and the Los Alamos W66. Both will enter the testing phase in October 1965, but in November 1968 the W65 will be discontinued in favor of the W66, which will continue testing in the late 1960s until it was entered in production in June 1974 as the first neutron bomb. Approximately 120 of these were produced, of which 70 were active in 1975 and 1976 as part of the Safeguard Program. ${ }^{1}$ When that program was shut down, they were stored and finally "decommissioned" in the early 1980s (Cochran et al., 1987, 23). The development of warheads with enhanced radiation for Lance continued, but in the early 1970s it turned to use a modified version of the M70, the M70 Mod 3.The development was subsequently postponed by President Jimmy Carter in 1978 following protests against his administration's plans to deploy neutron warheads to ground forces in Europe. President Ronald Reagan will restart production in 1981 after the Soviet Union detonated the first similar bomb on November 17, 1978. In 1981, the Soviet Union resumed its propaganda campaign against the US neutron bomb following Reagan's announcement to restart production. In 1983, Reagan announced a Strategic Defense Initiative that outpaced the production of the neutron bomb with vision and ambition, and the neutron bomb quickly faded from the center of public attention.

Three types of reinforced radiation weapons have been deployed by the United States: a) The W66 warhead for the Sprint anti-ICBM missile system, deployed in 1975 and withdrawn the following year along with the missile system; b) The medium-range W70 Mod 3 warhead developed for the MGM-52 Lance tactical missile; and c) W79 Mod 0 which was developed for nuclear artillery shells. The last two types were withdrawn by President George W. Bush in 1992, with the end of the Cold War (Christopher, 1997). The last W70 Mod3 warheads were disassembled in 1996, and the last W79 Mod 0 was reportedly

\footnotetext{
${ }^{1}$ The Safeguard program was a US anti-ballistic missile system (ABM) designed to protect US Air Force MCBM „Minuteman“ silos from attack, which would save the US nuclear deterrence fleet. The intention was primarily to protect from the very small Chinese MCBM fleet, as well as from limited attacks by the Soviet Union and various other scenarios with limited launch. A full attack by the Soviets would easily overwhelm the program. It was designed to allow for gradual upgrades to provide similar easy coverage across the United States over time.
} 
disassembled in 2003, which reportedly ended the disassembly of all W79 variants (NNSA, 2003). According to the official COX report, the United States has never deployed neutron weapons. But in a 1999 statement, Cohen suggested that the report play on definitions, in the sense that until US bombs were never deployed in Europe, they remained stockpiled in the United States (Cohen, 1999).

In addition to the two superpowers, France and China are known to have tested neutron or amplified radiation bombs, but neither country has decided to deploy neutron bombs. France conducted an early test of the technology in 1967 and a test of the current neutron bomb in 1980 (BBC Online Network, 1999). China conducted a successful test of the neutron bomb principle in 1984 and a successful neutron bomb test in 1988, although Chinese scientists said before 1988 that China did not need a neutron bomb, but developed it to serve as a "technological reserve" in case of need in the future (Ray, 2015).

In August 1999, the Indian government also announced that India was capable of producing neutron weapons (Karp, 1999).

The only country that is definitely known to have deployed dedicated neutron warheads at any time is Russia, which inherited from the USSR the ABM-3 Gazelle missile program equipped with neutron warheads. The ABM system contains at least 68 neutron warheads with a capacity of $10 \mathrm{KT}$ each and has been in service since 1995, with inertial missile testing every other year since 2014. The system is designed to destroy incoming nuclear warheads aimed at Moscow and other targets at the endoatmospheric level and is the lowest / last umbrella of the anti-ballistic missile system A-135 i.e. ABM in NATO terminology (Pike, 2015).

According to Mordechai Vanunu, Israel has been mass-producing neutron bombs since 1984 (Reed and Stillman, 2010, 181).

Significant controversy erupted in the United States and Western Europe in June 1997 after an report in the Washington Post describing US plans to equip the U.S. Air Force with neutron bombs. The article focused on the fact that it is the first weapon with a specific intent to kill people with radiation (Auten, 2008). Director of the National Laboratory Lawrence Livermore, Harold Brown, and Soviet Secretary-General Leonid Brezhnev both described the neutron bomb as a capitalist bomb because of its design to destroy people while preserving property (Herken, 2003, 332). Hence the sarcastic name of the neutron weapon as a "human weapon", due to the logic behind the intention to use it economically, i.e. not to cause unnecessary material damage in a destructive process in which only the living force suffers due to increased neutron action, but not the material means and technique. In addition to such views is the statement of the inventor of the neutron bomb Sam Cohen, in his intention to criticize the description of the W70 as a neutron bomb because that warhead can be configured to release 100 kilotons: "the W-70 ... is not even remotely a "neutron bomb." Instead of being the type of weapon that, in the popular mind, "kills people and spares buildings" it is one that both kills and physically destroys on 
a massive scale. The $\mathrm{W}-70$ is not a discriminate weapon, like the neutron bomb - which, incidentally, should be considered a weapon that "kills enemy personnel while sparing the physical fabric of the attacked populace, and even the populace too" (Cohen, 1999).

\section{Basic characteristics of neutron weapons}

Various claims about the construction of a neutron weapon have been circulating in the literature. The technical and technological solutions of the neutron weapon should meet various and even controversial requirements such as: the smallest possible critical mass of the lighter; limited energy of the lighter to the level necessary to start fusion; maximum weight reduction; fairly limited volume; longer retention of neutrons in the reaction zone, etc. There are the least dilemmas about thermonuclear reactions. Six types of thermonuclear reaction are possible, but the most likely is the fusion of hydrogen isotopes deuterium and tritium - which release energy of about 17.6 million electrovolts, with neutrons contributing about 80 percent (with a force of $14.1 \mathrm{MeV}$ ) while helium cores - alpha particles - contribute 20 percent $(3.5 \mathrm{MeV})$ of total energy released.

Compared to fission weapons, it should be borne in mind that fast neutrons in neutron (fusion) weapons qualitatively and quantitatively significantly exceed the neutrons that occur in the event of an explosion of fission missiles with the same force, thus extending over greater distances and causing major consequences. During the conceptualization, development and production of neutron weapons, efforts were made to make the radius of the average lethal dose as high as possible, minimizing mechanical-thermal action and additional radiological contamination. ${ }^{2}$

The most effective way to use a neutron weapon is an air nuclear blast, analogous to the use of small and very small nuclear weapons. The basic tools for using a neutron weapon are the same as for a nuclear weapons of small and very small fusion force. These are: short-range and medium-range missiles, artillery, tactical aircraft, and in some cases nuclear mines - fugues and sabotage devices. As early as the early 1980s, intensive work was done to improve some of the operational-tactical and constructive features of certain artillery pieces and to equip them to use these missiles, especially those of a very small force. The literature highlights the role and capability of the Lance missile systems, the M-110 and M-115 self-propelled howitzers with a caliber of $203.1 \mathrm{~mm}$ and the M-114A howitzer with a caliber of $155 \mathrm{~mm}$ - for the successful application of neutron weapons of small and very small force. Some sources claim that the

\footnotetext{
${ }^{2}$ For example, for a neutron missile with a force of $1 \mathrm{KT}$, that condition is optimally satisfied if the center of the explosion is at an altitude of 100 to 300 meters above flat ground. At those heights, the radius of the average lethal dose in a blast of this missile is 800 to 1000 meters.
} 
neutron flux attack was also the main goal for the design of various anti-aircraft weapons, such as the AIM-26 Falcon and CIM-10 Bomarc. An F-102 pilot noted: "GAR-11/AIM-26 was primarily a weapon-killer. The bomber(s, if any) was collateral damage. The weapon was proximity-fused to ensure detonation close enough so an intense flood of neutrons would result in an instantaneous nuclear reaction (NOT full-scale) in the enemy weapon's pit; rendering it incapable of functioning as designed...[O]ur first "neutron bombs" were the GAR11 and MB-1 Genie "(Maloney, 2014). Among other things, in the last decade of the Cold War, work has been done to modify some of the existing artillery weapons for the alternative use of classical and nuclear missiles.

\section{Some motives for the development and production of neutron weapons}

There has been a period in the doctrines of nuclear states, when the possibility of waging war and armed struggle has often been considered, with the use of tactical nuclear weapons involving small and very small neutron weapons. In a situation of assessing possible conflicts, local and limited wars have often been mentioned. It still seems that these wars, along with some new ones, are still the basic form in the doctrines of the use of force as a means of politics. The experiences from wars in which the focus was on the forms and methods of special warfare, have significantly changed the former doctrines of local wars, which in turn has the great powers to avoid those local wars that do not provide a sure and quick victory, i.e. to avoid direct engagement of its own armed forces. But if direct armed conflict could not be avoided, the technological elements of war would certainly have an advantage. In one such scenario, the significance of the "clean bomb" is stated in the option of eliminating additional contamination and minimizing the impact and heat effect, which would in some way erase or reduce the boundary between conventional and nuclear war, with all the consequences of military, political and international law.

During the Cold War, some of the doctrines of nuclear power considered the possibility of local wars with limited (selective) use of nuclear weapons, referring to limited use of tactical nuclear weapons. In such doctrines, a limited war, especially from the point of view of nuclear weapons, is all that is below the deterrent threshold, i.e. it comes down to the use of those weapons that will not cause the escalation of nuclear weapons of medium and great strength, and thus world nuclear war.

\footnotetext{
${ }^{3}$ Why is it called a clean bomb ?! Because leaves "no" additional harmful effects on the environment. In the case of a fission missile with the same force, the energy distribution is as follows: $50 \%$ mechanical; $33 \%$ thermal; $6 \%$ prompt radiation of which $2.5 \%$ is manifested in the form of neutron radiation, and 3.5\% gamma radiation; and $11 \%$ additional radiological contamination from fission fragments, primarily in the form of beta and gamma radiation. In (neutron) weapons with enhanced radiation, i.e. clean bomb, the energy distribution is as follows: $30 \%-40 \%$ mechanical; $20 \%-25 \%$ thermal; $30 \%-45 \%$ instantaneous radiation; and only $5 \%$ additional radiological contaminatioH.
} 
One of the motives for the production of neutron weapons should be considered from the position of negotiations between the nuclear powers in the amount of strategic nuclear weapons. The SALT agreements temporarily has limited the number of strategic nuclear weapons, but has provided a significant opportunity to continue a partially quantitative and almost unlimited qualitative race in strategic nuclear weapons in various directions. Each side sought to present as many nuclear weapons as possible from the middle and large strength of the existing arsenal as tactical, and among other things worked to transform certain forms of nuclear weapons with great strength into tactical ones.

One of the possible motives for the quantitative and quantitative improvement of tactical nuclear weapons is in the realm of technological warfare. Today's decisive technological warfare has emerged as an alternative to destructive warfare. The assumptions were that in the mutual calculation of that time superpowers, the focus must be on achieving technical and thus economic superiority. Instead of directly destroying the enemy's potential, it sought to obsolescence it permanently, forcing the enemy to incur constant and increasing costs or to reorient and engage in the development of other weapons, which would eventually make him economically weak.

In the professional literature of the West, in addition to the motive for the development of neutron weapons in that time, the inferiority of the United States and NATO to the USSR and the Warsaw Pact in human potential and conventional armaments is emphasized, especially in armored mechanized units. The projected preparation of high-powered nuclear missiles in combat operations in urban Europe, brings unwanted destruction to its own territory with a pronounced mechanical, thermal and additional radioactive action, which inevitably causes significant losses to its own population. Therefore, in their opinion, it was necessary to develop means that would have an effective destructive effect on people outside vehicles, in combat and non-combat vehicles, assets and facilities, while eliminating or minimizing unwanted destruction, fire and additional contamination.

\section{Conclusion}

The post-World War II era is characterized by vast scientific advances in the research, development, and improvement of nuclear weapons. In that period, the world's nuclear potential - with the introduction of new types of nuclear weapons that differ in construction, mode of action, strength and mode of launch - became more complex and diverse. Three generations can be distinguished in the development of small and very small tactical nuclear weapons. The third generation in the development of tactical nuclear weapons are missiles with small and very small force on the fusion principle of energy release, ie nuclear weapons with increased radiation action, the so-called neutron weapons. Three types of enhanced radiation weapons were deployed by the Unit- 
ed States: a) The W66 warhead for the Sprint anti-ICBM missile system, which was deployed in 1975 and withdrawn the following year along with the missile system; b) The W70 Mod 3 medium-range warhead developed for the MGM-52 Lance tactical missile; and (c) W79 Mod 0 which was developed for nuclear artillery shells. The only country that is known to have deployed dedicated neutron warheads at any given time is Russia, which inherited the USS-Gazelle missile program equipped with neutron warheads from the USSR. In addition to the two world superpowers, France, China, India and Israel have also tested neutron weapons.

Harold Brown and Leonid Brezhnev both described the neutron bomb as a capitalist bomb, designed to destroy people while preserving property. During the conceptualization, development and production of neutron weapons, efforts were made to make the radius of the average lethal dose as high as possible, by minimizing the mechanical-thermal action and the additional radiological contamination.

The motives for the development, improvement and production of small and very small nuclear weapons, as well as their possible application, must be considered in relation to the question: how and to what extent were these weapons in the service of politics? In response, two conclusions are drawn: 1) nuclear potential has proved to be a rational and effective means of strategy in relation to the opposing bloc, which has led to rational political, military-strategic and other relations between the great powers and reflected the wider world community; and 2) nuclear potential has not been shown to be sufficiently effective in its policy toward other countries, especially Third World countries, so, among other things, the emphasis was put on neutron weapons.

\section{References:}

AutEN, B., J. (2008). Carter's conversion: the hardening of American defense policy. University of Missouri Press.

BBC Online Network. (1999). Neutron bomb: Why 'clean' is deadly. BBC News. Available at: http://news.bbc.co.uk/2/hi/science/nature/395689.stm [Accessed 12Feb.2020].

Cochran, T., ARKIN, W., AND HoENIG, M. (1987). Nuclear Weapons Databook. In: U.S. nuclear warhead production. Volume 2. Ballinger Publishing.

CoHen, S. (1999). Check Your Facts: Cox Report Bombs. Insight on the News. Available at: https://www.questia.com/read/1G1-55426724/check-yourfacts-cox-report-bombs [Accessed 8 Jan. 2020].

HERKEN, G.(2003). Brotherhood of the Bomb: The Tangled Lives and Loyalties of Robert Oppenheimer, Ernest Lawrence, and Edward Teller. Macmillan. 
KARP, J. (1999). India Discloses It Is Able To Build a Neutron Bomb. The Wall Street Journal.

Available at: https://www.wsj.com/articles/SB934836102919955535 [Accessed 10Mar. 2020].

MALONEY, S. (2014). Secrets of the BOMARC: Re-examining Canada's Misunderstood Missile- Part 2. RCAF Journal, 2014,Volume 3, Issue 4.

Available at: http://www.rcaf-arc.forces.gc.ca/en/cf-aerospace-warfarecentre/elibrary/journal/2014-vol3-iss4-08-secrets-of-the-bomarc-part2.page\#note47 [Accessed 08 May. 2020].

McFADDEN, R., D. (2010). Samuel T. Cohen, Neutron Bomb Inventor, Dies at 89. The New York Times.

Available at: https://www.nytimes.com/2010/12/02/us/02cohen.html?pa gewanted=all [Accessed 18 Jan. 2020].

NNSA. (2003). NNSA Dismantles Last Nuclear Artillery Shell.(PDF). National Nuclear Security Administration.

Available at: https://web.archive.org/web/20111023163331/http:/nnsa. energy.gov/sites/default/files/nnsa/news/documents/PR_NA-03-16_W76Dismantled-LastNuclearArtilleryShell.pdf [Accessed 23 Jan. 2020].

PIKE, J (2015). 53T6 Gazelle.Globalsecurity.org. Available at:https://www.globalsecurity.org/wmd/world/russia/gazelle.htm [Accessed 01Apr. 2020].

RAY, J. (2015). Red China's "Capitalist Bomb": Inside the Chinese Neutron Bomb Program.(PDF). In: China Strategic Perspectives. Volume 8. INSS.

Available at: https://inss.ndu.edu/Portals/68/Documents/stratperspective/china/ChinaPerspectives-8.pdf [Accessed 03 Mar. 2020].

Reed, C., T. And Stillman, B.,D. (2010). The Nuclear Express: A Political History of the Bomb and Its Proliferation. Zenith Press, First edition. 